\title{
Resolution Influence on 3D Anthropometric Data Clustering for Fitting Design
}

\author{
Jianwei NIU ${ }^{1}$, Zhizhong LI $^{2 *}$ and Gavriel SALVENDY ${ }^{2,3}$ \\ ${ }^{1}$ School of Mechanical Engineering, University of Science and Technology, Beijing 100083, China \\ ${ }^{2}$ Department of Industrial Engineering, Tsinghua University, Beijing 100084, China \\ ${ }^{3}$ School of Industrial Engineering, Purdue University, West Lafayette, IN 47907, USA
}

Received May 7, 2008 and accepted March 30, 2009

\begin{abstract}
Sizing based on 3D anthropometric data may lead to significant improvement in fitting comfort of wearing products. However, the required computational load is a common problem in 3D data processing. In a previous study, wavelet analysis was adopted to establish a multiresolution description of 3D anthropometric data to reduce computational load and modeling complexity. K-means clustering was subsequently performed on the decomposed 3D samples. This study further examines the influence of decomposition level on clustering results. As a case study, 378 face samples, 447 head samples and 432 upper head samples were analyzed. Cluster membership variation on five different resolution levels was examined by using Cluster Membership Accuracy Rate (CMAR), which denotes the clustering consistency on the decomposed levels compared with the clustering results on the original data sets. For the face data sets, the CMAR values on the five decomposition levels are 100, 99.21, 97.88, 93.92 and $93.39 \%$, respectively; for upper heads, the CMAR values are 99.3, 99.1, 98.4, 92.1 and 84.3\%, respectively; while for whole heads, the CMAR values are 99.3, 98.2, 95.1, 85.5 and 77.9\%, respectively. These results indicate that clustering on the third decomposition level is proper for face and head scans in reducing computational load while maintaining at least $95 \%$ clustering accuracy.
\end{abstract}

Key words: Three dimensional (3D) anthropometry, Population fitting design, Multi-resolution, Clustering, Sizing

\section{Introduction}

Considerable attentions have been paid to proper fit of wearing products for a long time ${ }^{1-7)}$. For wearing products which need close fitting, such as helmet, goggle, face mask, spectacles or shoes, they must be designed to well match the human body surfaces of the users. For example, according to a survey of wearing comfort and fit issue of oxygen masks (derived from the Soviet mask system in the 1950s) among 213 Chinese fighter pilots ${ }^{8}$, nearly $80 \%$ pilots considered the masks did not fit their faces, and $70 \%$ pilots felt masks squeezed their noses and faces, inconvenient to don, doff or adjust. For another example, studies on fitting issue of shoes show that poor fit has been the main source of causing blistering, chafing,

*To whom correspondence should be addressed.

E-mail: zzli@tsinghua.edu.cn black toes, bunions, pain, and tired feet ${ }^{9)}$.

Sizing tariff is one of the most important factors influencing the fit and performance of products ${ }^{10,11)}$. Some linear measurements were usually used as key dimensions in traditional sizing methods ${ }^{12-14)}$. For example, the current used oxygen mask sizing system for fighter pilots in China is based on the fighter pilots' morphological facial length and bizygomatic breadth or mouth breadth ${ }^{15)}$. For helmet sizing, head length and head breadth were usually adopted as the key dimensions to group the samples $^{16,17)}$. However, if only one or two dimensions are taken into account, geometric characteristics and internal structure of human surface are not adequately considered. This may lead to design deficiency on fitting comfort. The demand for new methods arose when traditional sizing systems proved to be inadequate for current craniofacial design problems.

3D anthropometry surveys, such as the CAESAR pro- 
ject $^{18)}$, SizeUK ${ }^{19)}$, SizeUSA (www.tc2.com), SizeChina (www.sizechina.com), etc., can provide tremendous amount of shape information of the human body. Researchers have utilized 3D anthropometric data in the design of helmet ${ }^{20)}$, footwear ${ }^{3)}$, human-harness ${ }^{5)}$ and hand tools $^{21)}$, etc. However, there exist some difficulties such as high computational load and modeling complexity when using 3D anthropometric data in product design. Multi-resolution can be used to establish a flexible model of 3D data, and then enable data manipulation and analysis progressively in a coarse-to-fine fashion ${ }^{22)}$. In our previous studies ${ }^{23-26)}$, wavelet analysis was adopted to establish a multi-resolution description of 3D anthropometric data to reduce computational load and modeling complexity and K-means clustering was subsequently performed on block distance based vectors of decomposed 3D samples to segment a population into groups according to size and shape. This study further examines the influence of decomposition level on clustering results in the case study of upper head, face and whole head samples, and report findings from this case study.

\section{Methodology}

\section{Raw Data}

The raw data for the case study include head cross sections scans of 510 young male Chinese soldiers (aged from 19 to 23) collected by a military department in 2002 . The point number of one head sample varies from minimum 38,826 to maximum 56,200 with an average of 46,400 and standard deviation of 3,117. Noise data on each cross section have been removed manually by visual check under CAD software. The face surfaces were spanned from the whole heads by the front $\pi / 2$ wedge angle along a line going through the centroid and pointing to the top of a head. Due to alignment, some unsuitable samples were removed. Therefore, only 378 face samples, 447 whole head samples and 432 upper head samples were derived from the 510 raw data sets and further analyzed.

\section{Multi-resolution representation of $3 D$ surface}

Wavelet decomposition was adopted to establish a multi-resolution representation of the 3D data (please refer to Niu et al. ${ }^{24)}$ and Niu et al. ${ }^{25)}$ for details). The lower resolution part preserves the major information and describes the main shape, while ignores the inappreciable geometric characteristics. By using the lower resolution part instead of the original data sample, shape analysis and comparison can be accomplished to segment the population according to their 3D shape. This model also can help the engineers select appropriate resolution level to customize the wearing product based on specific fitting requirements.

In the case study, each 3D sample was decomposed under five levels resulting in 3D surfaces with 4,489, $1,225,361,121$, and 49 control points respectively. From the first to the fifth levels, the number of control points decreases but the major shape of a surface remains with different levels of details.

\section{Block distance-based vector descriptor for sample sur-} faces

A block distance-based vector descriptor was used for shape clustering. We used the inscribed surface of all the samples as the reference surface. The inscribed surface and all sample surfaces were divided into a predefined number of blocks. The distance between two corresponding blocks on a sample surface $i$ and the inscribed surface, namely $S(i)$, can be constructed with two parts, namely $S_{1}(i)$ and $S_{2}(i)$, as

$$
S_{1}(i)=\sum_{j=1}^{n_{i}} \operatorname{dis}\left(p_{i, j}\right), i=1,2,3, \ldots, m
$$

where $p_{i, j}$ is the $j$ th point, and $n_{i}$ represents the number of points falling into the $i$ th block, and $\operatorname{dis}\left(p_{i, j}\right)$ denotes the Euclidean distance between the corresponding points. $S_{2}(i)$ can be calculated as

$$
S_{2}(i)=\sum_{j=1}^{n_{i}}\left|\operatorname{dis}\left(p_{i, j}\right)-\frac{S_{1}(i)}{n_{i}}\right|, i=1,2,3, \ldots, m
$$

$S_{2}$ describes the geometric variation in the corresponding local areas.

From the definitions it can be seen that $S_{1}(i)$ s mainly reflect size difference of two paired blocks, while $S_{2}(i) \mathrm{s}$ mainly reflect the local shape difference. In such a way, the 3D geometry of a surface can be characterized by a vector $\left(S_{1}(1), S_{2}(1), S_{1}(2), S_{2}(2), \ldots, S_{1}(m), S_{2}(m)\right)$.

\section{K-means clustering}

$\mathrm{K}$-means clustering was adopted in this study to classify the population into groups. For k-means clustering, the number of the clusters should be specified beforehand. Currently there are three sizes of oxygen masks in China, i.e., small, medium and large. Wang and Yuan ${ }^{15)}$ presented a new oxygen mask sizing system where they partitioned the Chinese face samples into four sizes, namely small size, medium-narrow size, medium-wide size and large size. The latest Chinese national standard of 3D craniofacial dimensions for male soldiers partitioned the craniofacial data sets into seven standard head models according to their shapes. In order for the ease of comparison, the number of $\mathrm{K}$ for the clustering on face data sets was set as four while that for the clustering on whole head and upper head data sets was set as seven. 
Clustering evaluation and cluster membership accuracy rate

K-means clustering based on block distance vectors (If not specially mentioned, block distance were normalized) was applied to the data sets on different decomposition levels and also on original data sets. A criterion for clustering evaluation in this study is size-weighted variance. The size-weighted variances were calculated by pooling the within size variance of block distances. It can be defined as follows,

$$
\text { SizeWeightedVar }=\frac{1}{N} \sum_{i=1}^{k} n_{i} \times \operatorname{var}(x)_{x \in C_{i}}
$$

where $k$ is the number of clusters, $N$ is the number of the samples, $n_{i}$ is the number of samples in the $k$ th cluster, $C_{i}$ denotes the $k$ th cluster, $\operatorname{var}(x)$ denotes the variance of the samples belonging to the cluster $C_{i}$. From the definition, a lower value of the size-weighted variance means better clustering.

Besides the size-weighted variances, Cluster Membership Accuracy Rate (CMAR) with respect to various decomposition levels compared to the original surface is also addressed to evaluate the resolution influence on $3 \mathrm{D}$ anthropometric data clustering for fitting design. Given the result of clustering on the original data sets as the reference, CMAR of a resolution level $i$ can be defined as follows,

$$
C_{M A R_{i}}=\frac{N_{i}}{N}(i=0,1,2,3,4,5)
$$

where $N_{i}$ is the number of samples correctly grouped when compared to the clustering result of the original data sets, and $N$ is the total sample number.

\section{Results and Discussions}

Multi-resolution representation and block division

Multi-resolution representation and block division of
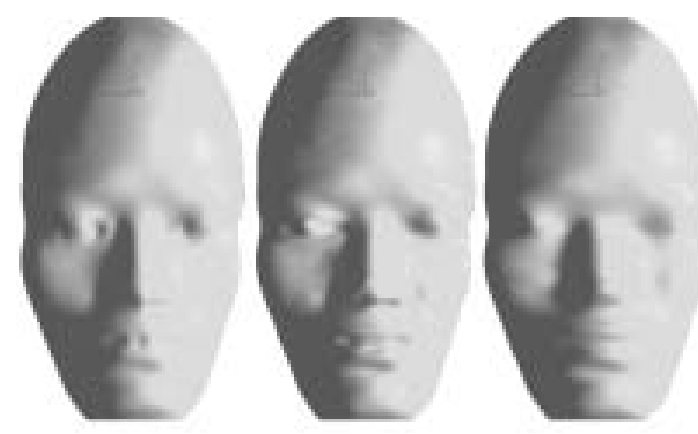

(a) (c)

face samples, upper head samples and whole head samples were implemented. The result of a face sample is illustrated in Fig. 1, where (a) represents the original face surface, and (b) to (f) correspond to the decomposed face surfaces on the decomposition level of one, two, three, four and five respectively. Quantitative approximation errors were investigated by Niu et al. ${ }^{25}$ ) with respect to various decomposition levels compared to the original surfaces showing that the decomposed surfaces could preserve the main shape of the original ones before the decomposition level reaches five. For upper heads, the average approximation errors at the 2 nd and 3 rd decomposition levels are $0.172 \mathrm{~mm}$ and $0.457 \mathrm{~mm}$, respectively. In contrast, for faces, the corresponding values are $0.240 \mathrm{~mm}$ and $0.702 \mathrm{~mm}$, respectively. For the design of products such as helmets, the approximation error of upper head surfaces at the 3rd decomposition level may be acceptable, while for the design of products such as goggles and respirators, the approximation error of face surfaces at the 2 nd decomposition level may be more suitable.

The block division result of a face is shown in Fig. 2 .

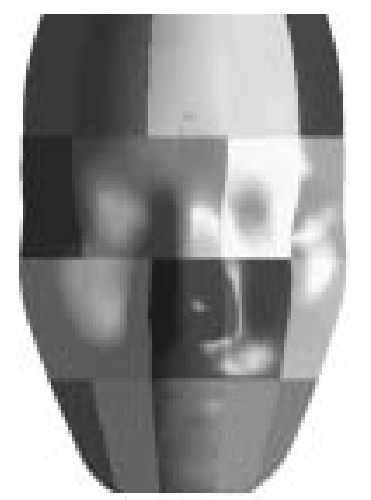

(a) front view

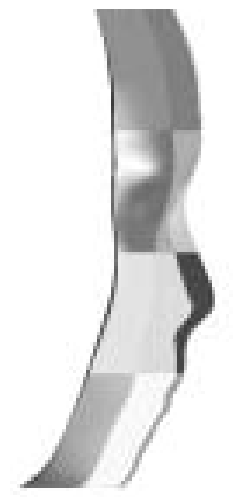

(b) side view

Fig. 2. Block division of a sample face.

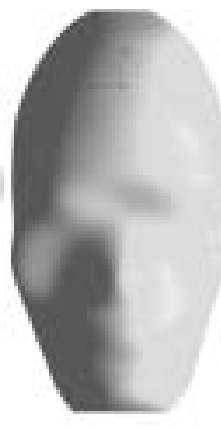

(d)

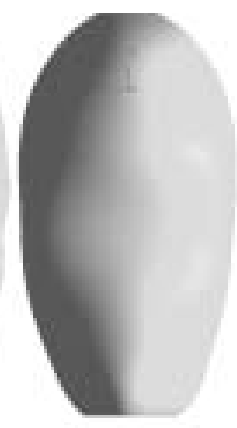

(e)

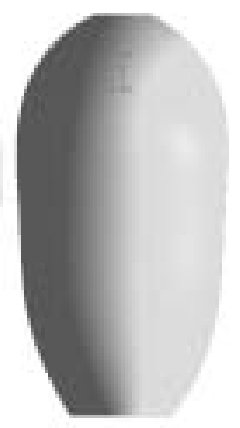

(f)

Fig. 1. Multi-resolution model of a face sample (adapted from Niu et al. ${ }^{25}$ )). 
The block division was evenly made along the height and span angle for the studied surfaces, without considering anatomical correspondence. It can be seen that blocks on the forehead are regular patches, while blocks lying in the areas of eyes, nose and mouth are fluctuant and irregular, and their shapes vary evidently between each other. Blocks on the chin area are moderately regular.
Sizing results

Table 1 shows the size-weighted variances of the variables of clustering on different resolutions for faces. Lower values of the size-weighted variances mean that the spread degree of the block distances is smaller. As shown in Table 1, the ratios of size-weighted variances are all equal to and close to 1 although some limited variations can be observed. This may imply that the clustering results under different decomposition levels are

Table 1. Size-weighted variances for faces on different decomposition levels ( $N=378,20$ blocks each face)

\begin{tabular}{|c|c|c|c|c|c|c|c|}
\hline \multirow[b]{2}{*}{ variable } & \multicolumn{4}{|c|}{ Size-weighted variances } & \multicolumn{3}{|c|}{ Ratio of size-weighted variances } \\
\hline & Original & Level 1 & Level 3 & Level 5 & $\begin{array}{c}\text { Level } 1 \\
\text { vs. } \\
\text { original }\end{array}$ & $\begin{array}{c}\text { Level } 3 \\
\text { vs. } \\
\text { original }\end{array}$ & $\begin{array}{c}\text { Level } 5 \\
\text { vs. } \\
\text { original }\end{array}$ \\
\hline$S_{1}(1)$ & 0.53 & 0.53 & 0.52 & 0.52 & 1 & 0.98 & 0.98 \\
\hline$S_{1}(2)$ & 0.46 & 0.46 & 0.46 & 0.51 & 1 & 1 & 1.11 \\
\hline$S_{1}(3)$ & 0.47 & 0.47 & 0.47 & 0.53 & 1 & 1 & 1.13 \\
\hline$S_{1}(4)$ & 0.43 & 0.43 & 0.43 & 0.47 & 1 & 1 & 1.09 \\
\hline$S_{1}(5)$ & 0.48 & 0.48 & 0.47 & 0.47 & 1 & 0.98 & 0.98 \\
\hline$S_{1}(6)$ & 0.34 & 0.34 & 0.34 & 0.36 & 1 & 1 & 1.06 \\
\hline$S_{1}(7)$ & 0.35 & 0.35 & 0.34 & 0.32 & 1 & 0.97 & 0.91 \\
\hline$S_{1}(8)$ & 0.33 & 0.33 & 0.33 & 0.32 & 1 & 1 & 0.97 \\
\hline$S_{1}(9)$ & 0.36 & 0.36 & 0.34 & 0.31 & 1 & 0.94 & 0.86 \\
\hline$S_{1}(10)$ & 0.33 & 0.33 & 0.33 & 0.36 & 1 & 1 & 1.09 \\
\hline$S_{1}(11)$ & 0.27 & 0.27 & 0.27 & 0.27 & 1 & 1 & 1 \\
\hline$S_{1}(12)$ & 0.23 & 0.23 & 0.23 & 0.23 & 1 & 1 & 1 \\
\hline$S_{1}(13)$ & 0.27 & 0.27 & 0.26 & 0.25 & 1 & 0.96 & 0.93 \\
\hline$S_{1}(14)$ & 0.24 & 0.24 & 0.24 & 0.23 & 1 & 1 & 0.96 \\
\hline$S_{1}(15)$ & 0.28 & 0.28 & 0.28 & 0.27 & 1 & 1 & 0.96 \\
\hline$S_{1}(16)$ & 0.35 & 0.35 & 0.35 & 0.35 & 1 & 1 & 1 \\
\hline$S_{1}(17)$ & 0.27 & 0.27 & 0.27 & 0.29 & 1 & 1 & 1.07 \\
\hline$S_{1}(18)$ & 0.25 & 0.25 & 0.25 & 0.27 & 1 & 1 & 1.08 \\
\hline$S_{1}(19)$ & 0.27 & 0.27 & 0.28 & 0.27 & 1 & 1.04 & 1 \\
\hline$S_{1}(20)$ & 0.36 & 0.36 & 0.37 & 0.36 & 1 & 1.03 & 1 \\
\hline$S_{2}(1)$ & 0.83 & 0.83 & 0.85 & 0.64 & 1 & 1.02 & 0.77 \\
\hline$S_{2}(2)$ & 0.68 & 0.68 & 0.64 & 0.57 & 1 & 0.94 & 0.84 \\
\hline$S_{2}(3)$ & 0.7 & 0.7 & 0.65 & 0.61 & 1 & 0.93 & 0.87 \\
\hline$S_{2}(4)$ & 0.64 & 0.64 & 0.62 & 0.59 & 1 & 0.97 & 0.92 \\
\hline$S_{2}(5)$ & 0.76 & 0.76 & 0.72 & 0.57 & 1 & 0.95 & 0.75 \\
\hline$S_{2}(6)$ & 0.7 & 0.7 & 0.64 & 0.68 & 1 & 0.91 & 0.97 \\
\hline$S_{2}(7)$ & 0.77 & 0.77 & 0.78 & 0.5 & 1 & 1.01 & 0.65 \\
\hline$S_{2}(8)$ & 0.64 & 0.64 & 0.58 & 0.61 & 1 & 0.91 & 0.95 \\
\hline$S_{2}(9)$ & 0.89 & 0.89 & 0.87 & 0.6 & 1 & 0.98 & 0.67 \\
\hline$S_{2}(10)$ & 0.85 & 0.85 & 0.8 & 0.8 & 1 & 0.94 & 0.94 \\
\hline$S_{2}(11)$ & 0.9 & 0.9 & 0.91 & 0.99 & 1 & 1.01 & 1.1 \\
\hline$S_{2}(12)$ & 0.65 & 0.65 & 0.66 & 0.68 & 1 & 1.02 & 1.05 \\
\hline$S_{2}(13)$ & 0.84 & 0.84 & 0.79 & 0.95 & 1 & 0.94 & 1.13 \\
\hline$S_{2}(14)$ & 0.74 & 0.74 & 0.73 & 0.72 & 1 & 0.99 & 0.97 \\
\hline$S_{2}(15)$ & 0.88 & 0.88 & 0.9 & 0.84 & 1 & 1.02 & 0.95 \\
\hline$S_{2}(16)$ & 0.86 & 0.86 & 0.84 & 0.84 & 1 & 0.98 & 0.98 \\
\hline$S_{2}(17)$ & 0.72 & 0.72 & 0.76 & 0.82 & 1 & 1.06 & 1.14 \\
\hline$S_{2}(18)$ & 0.72 & 0.72 & 0.72 & 0.75 & 1 & 1 & 1.04 \\
\hline$S_{2}(19)$ & 0.79 & 0.79 & 0.84 & 0.86 & 1 & 1.06 & 1.09 \\
\hline$S_{2}(20)$ & 0.86 & 0.86 & 0.87 & 0.88 & 1 & 1.01 & 1.02 \\
\hline
\end{tabular}

$S_{1}(i)$ - the first part of block-based distance between two blocks; $S_{2}(i)$ - the second part of block-based distance between two blocks; $i$ - No. of a block. 
quite consistent. To further confirm it, CMAR values should be examined.

Given the result of clustering on the original data sets as the reference, cluster membership variation on different resolution level of each sample was investigated by using the CMAR, as summarized in Table 2. For the face data sets, the numbers of improper clustering samples on the first, second, third, fourth and fifth decomposition levels are $0,3,8,23$ and 25 respectively (sample size is 378). CMAR values on the five decomposition levels are 100, 99.21, 97.88, 93.92 and $93.39 \%$ respectively. These values are very high. For the upper head data sets, the numbers of improper clustering samples on the first, second, third, fourth and fifth decomposition levels are 3, 4, 7, 34 and 68 respectively (sample size is 432). CMAR values on the five decomposition levels are 99.3, 99.1, 98.4, 92.1 and $84.3 \%$ respectively. That is, until the fifth decomposition level CMAR drops to be lower than $90 \%$. As for whole head data sets, the numbers of improper clustering samples on the first, second, third, fourth and fifth decomposition levels are 3, 8, 22, 65 and 99 respectively (sample size is 447 ), corresponding to CMAR val- ues of 99.3, 98.2, 95.1, 85.5 and $77.9 \%$ respectively, showing a more quick CMAR drop with decomposition level, but CMAR values until decomposition level 3 are still very high $(>95 \%)$, although the control point number has dropped from 4,489 to 361 . Therefore, such high CMAR values indicate that wavelet decomposition level has inconspicuous influence on the shape clustering before the decomposition level reaches four. This strengthens the conclusion made by Niu et al. ${ }^{24)}$ and Niu et al. ${ }^{25)}$ that data set at a lower resolution can preserve the major information and describes the main shape of the original 3D form. To sum up the previous analysis, as for 3D craniofacial data sets, $\mathrm{k}$-means clustering on the decomposed data set other than on the original one can likewise result in appropriate sizing for the purpose of population fitting design.

The above discussion is based on a practical viewpoint and related standards. The most common accommodation rates adopted in product design are $90 \%$ and $95 \%$. We may say $92.1 \%$ differs from $84.3 \%$ since there is greater difference between them and $84.3 \%$ is lower than $90 \%$. On the contrast, it's not meaningful to distinguish

Table 2. Cluster membership statistics

\begin{tabular}{|c|c|c|c|c|c|c|}
\hline \multirow{2}{*}{ Cluster ID } & \multicolumn{6}{|c|}{ Cluster size } \\
\hline & Original & Level 1 & Level 2 & Level 3 & Level 4 & Level 5 \\
\hline \multicolumn{7}{|c|}{ Face data sets, $N=378,20$ blocks each sample } \\
\hline 1 & 52 & 52 & 52 & 52 & 51 & 59 \\
\hline 2 & 60 & 60 & 59 & 57 & 54 & 55 \\
\hline 3 & 136 & 136 & 135 & 137 & 143 & 134 \\
\hline 4 & 130 & 130 & 132 & 132 & 130 & 130 \\
\hline Improper clustered samples & $\ldots$ & 0 & 3 & 8 & 23 & 25 \\
\hline CMAR $(\%)$ & $\ldots$ & 100 & 99.21 & 97.88 & 93.92 & 93.39 \\
\hline \multicolumn{7}{|c|}{ Upper head data sets, $N=432,20$ blocks each sample } \\
\hline 1 & 92 & 92 & 92 & 91 & 94 & 91 \\
\hline 2 & 55 & 55 & 55 & 56 & 55 & 52 \\
\hline 3 & 82 & 81 & 81 & 83 & 80 & 91 \\
\hline 4 & 35 & 35 & 34 & 34 & 33 & 31 \\
\hline 5 & 68 & 70 & 71 & 69 & 70 & 76 \\
\hline 6 & 35 & 34 & 34 & 35 & 36 & 35 \\
\hline 7 & 65 & 65 & 65 & 64 & 64 & 56 \\
\hline Improper clustered samples & $\ldots$ & 3 & 4 & 7 & 34 & 68 \\
\hline CMAR $(\%)$ & $\ldots$ & 99.3 & 99.1 & 98.4 & 92.1 & 84.3 \\
\hline \multicolumn{7}{|c|}{ Whole head data sets, $N=447,20$ blocks each sample } \\
\hline 1 & 61 & 60 & 61 & 60 & 57 & 56 \\
\hline 2 & 57 & 57 & 58 & 55 & 64 & 65 \\
\hline 3 & 53 & 53 & 54 & 55 & 55 & 39 \\
\hline 4 & 91 & 91 & 88 & 87 & 79 & 94 \\
\hline 5 & 57 & 57 & 59 & 63 & 70 & 75 \\
\hline 6 & 34 & 34 & 34 & 33 & 32 & 35 \\
\hline 7 & 94 & 95 & 93 & 94 & 90 & 83 \\
\hline Improper clustered samples & $\ldots$ & 3 & 8 & 22 & 65 & 99 \\
\hline CMAR $(\%)$ & $\ldots$ & 99.3 & 98.2 & 95.1 & 85.5 & 77.9 \\
\hline
\end{tabular}

CMAR - Cluster Membership Accuracy Rate. 
93.9\% and $93.4 \%$, since the difference is so small and both are higher than $90 \%$. As to related standards, taking respirator as an example, annual fit testing is required under OSHA's respirator standard 29 CFR 1910.13427) and recommended by the ANSI guidelines for respiratory protection (ANSI Z88.2-1992) ${ }^{28}$. Respirators designed to fit these panels were expected to accommodate at least $95 \%$ of the wearers. Considering the contact interfaces of such kind of products would have a certain level of adjustability and flexibility, a little lower than $95 \%$ can be considered for judgment of acceptable CMAR since it is based on rigid surface analysis.

Average sample of a cluster can be used as the representative sample of the cluster. It can be generated by averaging coordinates of corresponding points of all the samples belonging to the same cluster. Taking face data sets as an example, different views of the merged average face samples of the clusters $(N=378)$ on different decomposition levels are shown in Fig. 3. The figure can be helpful to obtain an intuitive understanding of the clus-

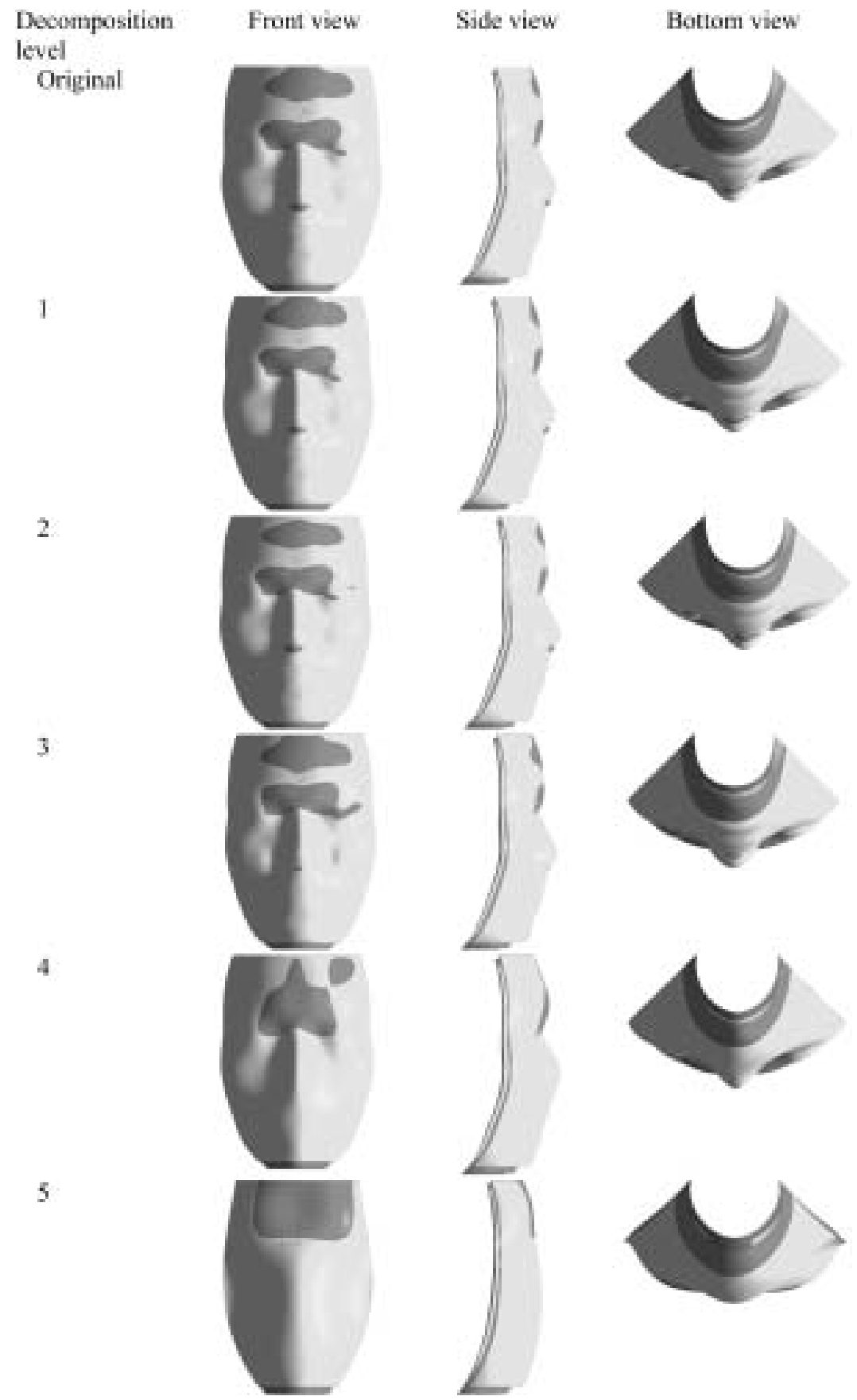

Fig. 3. Different views of the merged average faces of clusters $(N=378)$. 
tering results and the shape dissimilarity among clusters.

\section{Conclusions}

In this paper, the influence of decomposition level on clustering results of 3D face data sets, upper head data sets and whole head data sets were studied. Cluster membership accuracy rates (CMARs) on different resolution levels, namely from the first to the fifth decomposition levels, were investigated. Results indicate that clustering on the third decomposition level is proper for face and head scans in reducing computational load while maintaining at least $95 \%$ clustering accuracy. High CMAR values imply that wavelet decomposition level does not have remarkable influence on shape clustering before the decomposition level reaches five. Considering the difference of the interface of different products with human body surface, engineers could be encouraged to adopt the CMAR value as a valuable reference to select a proper decomposition level for the design of a specific product based on the corresponding fitting requirement.

\section{Acknowledgements}

This work was supported by the National Natural Science Foundation of China (No.70571045).

\section{References}

1) Whitestone JJ, Robinette KM (1997) Fitting to maximize performance of HMD systems. In: Head-Mounted Displays: designing for the User, Melzer JE and Moffitt KW (Eds.), 175-206, McGraw-Hill, New York.

2) Harrison CA, Robinette KM (2005) Principles of fit to optimize helmet sizing. In: Strategies to Maintain Combat Readiness during Extended Deployments-A Human Systems Approach, 6.1-6.16, NATO Research and Technology Organization, Neuilly sur Seine, France.

3) Witana CP, Xiong SP, Zhao JH, Goonetilleke RS (2006) Foot measurements from three-dimensional scans: a comparison and evaluation of different methods. Int $\mathbf{J}$ Ind Ergon 36, 789-807.

4) $\mathrm{Au}$ EYL, Goonetilleke RS (2007) A qualitative study on the comfort and fit of ladies' dress shoes. Appl Ergon 38, 687-96.

5) Hsiao HW, Whitestone J, Kau TY (2007) Evaluation of Fall Arrest Harness Sizing Schemes. Hum Factors 49, 447-64.

6) Lee HY, Hong KH (2007) Optimal brassiere wire based on the 3D anthropometric measurements of under breast curve. Appl Ergon 38, 377-84.

7) Zhuang Z, Bradtmiller B, Shaffer RE (2007) New respirator fit test panels representing the current U.S. civilian work force. J Occup Environ Hyg 4, 647-59.

8) Wang XW, Yuan XG, Peng Y, Bai JX, Wang RD, Sun
MZ, Cheng LM (2001) Survey of dressing comfort and fit issue of oxygen masks and its human engineering significance. Chinese J Ergonom 3, 6-9 (in Chinese).

9) Rossi WA (1988) The futile search for the perfect shoe fit. J Test Eval 16, 393-403.

10) Liu BG, Li LM, Zhang JW, Ma XS, Cao BP (1999) Study on factors affecting the suitability of pressure oxygen mask. Acta Aeronaut Astronaut Sin 20 (Suppl), 47-8 (in Chinese).

11) Chung MJ, Lin HF, Wang MJ (2007) The development of sizing systems for Taiwanese elementary- and highschool students. Int J Ind Ergon 37, 707-16.

12) Gouvali MK, Boudolos K (2006) Match between school furniture dimensions and children's anthropometry. Appl Ergon 37, 765-73.

13) Zheng R, Yu W, Fan JT (2007) Development of a new Chinese bra sizing system based on breast anthropometric measurements. Int J Ind Ergon 37, 697-705.

14) Liu BS (2008) Incorporating anthropometry into design of ear-related products. Appl Ergon 39, 115-21.

15) Wang XW, Yuan XG (2001) Study on type and sizing tariff of aircrew oxygen masks. J Beijing Univ Aeronaut Astronaut 27, 309-12 (in Chinese).

16) Robinette KM, Whitestone JJ (1992) Methods for Characterizing the Human Head for the Design of Helmets. AL-TR-1 992-0061. Wright-Patterson AFB $\mathrm{OH}$ : Armstrong Laboratory.

17) Robinette KM, Whitestone JJ (1994) The need for improved anthropometric methods for the development of helmet systems. Aviat Space Environ Med 65, A95-99.

18) Robinette KM, Blackwell S, Daanen H, Fleming S, Boehmer M, Brill T, Hoeferlin D, Burnsides D (2002) CAESAR, Final Report, Volume I: Summary. AFRLHE-WP-TR-2002-0169. United States Air Force Research Lab, Human Effectiveness Directorate, Crew System Interface Division, Dayton Ohio.

19) London College of Fashion. SizeUK announce results from UK National Sizing Survey. http://www.fashion. arts.ac.uk/sizeuk.htm. Accessed March 30, 2009.

20) Meunier P, Tack D, Ricci A, Bossi L, Angel H (2000) Helmet accommodation analysis using 3D laser scanning. Appl Ergon 31, 361-9.

21) Rogers MS, Barr AB, Kasemsontitum B, Rempel DM (2008) A three-dimensional anthropometric solid model of the hand based on landmark measurements, Ergonomics 51, 511-26.

22) Garland M (1999) Multi-resolution modeling: survey \& future opportunities. In: Eurographics ' 99 - State of The Art Reports, Aire-la-Ville CH (Ed.), 111-31, European Association for Computer Graphics, Geneve.

23) Niu JW, Li ZZ, Salvendy G (2006) Multi-resolution description of 3D anthropometric data. The 16th Triennial Congress of the International Ergonomics Association (IEA 2006), July 10-14, Maastricht, the Netherlands.

24) Niu JW, Li ZZ, Salvendy G, (2007) A case study of 
multi-resolution representation of heads. In: Digital Human Modeling, HCII 2007, LNCS 4561, Duffy VG (Ed.), 171-8, Springer-Verlag, Berlin Heidelberg.

25) Niu JW, Li ZZ, Salvendy G (2009) Multi-resolution Description of Three-dimensional Anthropometric Data for Design Simplification. Appl Ergon 40, 807-10.

26) Niu JW, Li ZZ, Salvendy G (2009) Multi-resolution shape description and clustering of three-dimensional head data. Ergonomics 52, 240-64.

27) Occupational Safety and Health Administration (1998) 29 CFR 1910.134: Respiratory protection. http://www. ehso.com/OSHA1910_134.htm. Accessed June 29, 2008.

28) American National Standards Institute, Inc. (1992) ANSI Z88.2-1992: American National Standard for Respiratory Protection. 